ISSN-i 1390-7042 | ISSN-e 2602-8484

\title{
RECUPERACIÓN DE ORO MEDIANTE CONCENTRACIÓN GRAVIMÉTRICA UTILIZANDO CONCENTRADORES TIPO Z EN EL DISTRITO MINERO ZAMORA-ECUADOR
}

\section{Gold recovery by gravimetric concentration using Z-type concentrators in the Zamora-Ecuador mining district}

\begin{tabular}{ll}
\hline $\begin{array}{l}\text { Cristhian Feijoo Loayza } \\
\text { Instituto de Investigación Geológico y Energético } \\
\text { cristhian.feijoo@geoenergia.gob.ec } \\
\text { ORCID: https://orcid.org/0000-0003-1261-1768 }\end{array}$ & $\begin{array}{l}\text { Fausto Acosta Fiallos } \\
\text { Instituto de Investigación Geológico y Energético } \\
\text { fausto.acosta@geoenergia.gob.ec } \\
\text { orcID: https://orcid.org/0000-0001-6502-4378 }\end{array}$ \\
\hline
\end{tabular}

Recibido: 03 de junio de 2020 Aprobado: 18 de diciembre de 2020 ARTÍ́CULO ORIGINAL

DOI: $10.29166 /$ REVFIG.V1I2.2283
PAlABRAS Clave Concentrador gravimétrico tipo $\mathrm{z}$, oro fino, recuperación.

KEYWORDS Z-type gravimetric concentrator, fine gold, recovery.

\section{RESUMEN}

El Ecuador por su relieve geográfico tiene un alto potencial de recursos mineros, los cuales pueden ser explotados de manera adecuada para generar altos réditos económicos que beneficien al país. Muestra de ello es la provincia de Zamora Chinchipe, la cual contiene uno de los cinco principales yacimientos ecuatorianos de oro aluvial, donde se utilizan métodos de extracción gravimétricos. Sin embargo, también es evidente la falta de tecnificación en los procesos de beneficio de minerales en este sector minero. Por ejemplo, es muy común el uso del concentrador gravimétrico tipo z. Este tipo de equipo presenta una serie de inconvenientes durante su operación para la recuperación de mineral, por ejemplo, las distancias cortas que recorre el mineral en el lavado, el escape de oro libre fino por la corriente, el uso de sacos de yute en los canalones, etc. Para el presente estudio, se tomaron muestras de nueve plantas de beneficio del sector con el fin de determinar la eficiencia de recuperación de oro, se analizó la configuración de los diferentes concentradores tipo $\mathrm{z}$ estudiados, parámetros de operación, y la presencia de mercurio en las colas de descarga. Los resultados muestran que las recuperaciones de oro en las plantas estudiadas van desde el $12 \%$ al $98 \%$, demostrando que el equipo es ineficiente cuando el mineral presenta oro fino y, por lo tanto, es necesario implementar nuevas alternativas que mejoren los procesos de recuperación.

\footnotetext{
ABSTRACT

Ecuador has a high potential for mining resources, which can be properly exploited to generate high economic returns for the benefit of the country, proof of this is the province of Zamora Chinchipe which is one of the 5 main Ecuadorian alluvial gold deposits, where gravimetric extraction methods are used. However, it is also evident the lack of technification in the mineral profit processes in this mining sector. For example, the use of the Z-type gravimetric concentrator is very common. This type of equipment has several drawbacks during its operation for mineral recovery. For the present study, samples were taken from nine profit plants of the sector in order to determine the efficiency of recovery of gold, the configuration of the different Z-type concentrators studied, operating parameters was analyzed, and the presence of mercury in the tailings. The results show that gold recoveries in the plants studied range from $12 \%$ to $98 \%$, showing that the equipment presents disadvantages when the ore presents fine gold and therefore it is necessary to have alternatives that improve the recovery processes.
} 


\section{INTRODUCCIÓN}

La geología de Zamora-Ecuador consta de rocas, mesozoicas y cenozoicas de diversos ambientes genéticos. La geología de la faja subandina al sureste del Ecuador está dominada por la intrusión de batolitos tipo I, del arco continental Jurásico Zamora (complejo intrusivo de Zamora), que intruyen a rocas prejurásicas. Los volcánicos de la Fm. Misahuallí son la expresión extrusiva del arco continental Jurásico. Afloran rocas volcanoclásticas de la U. Piuntza y lavas de la U. Pachicutza (triásica) en ventanas tectónicas sobre el complejo intrusivo de Zamora. Las rocas sedimentarias de las formaciones Hollín y Napo de edad cretácica yacen discordantes sobre el complejo intrusivo de Zamora (INIGEMM, 2017).

Los placeres aluviales de oro se crean por la erosión y liberación de los depósitos hidrotermales de oro o vetas auríferas formadas, que a su vez son transportados por sistemas de corrientes de aguas abajo a distancias que van desde metros a cientos de kilómetros, como se indica en la figura 1 (Stewart et al., 2017; Marsden \& House, 2006).

La provincia de Zamora Chinchipe forma parte de uno de los cincos principales yacimientos ecuatorianos de oro aluvial originados a partir de rocas metamórficas de la cordillera Real, lo que ha permitido el surgimiento de la actividad minera para recuperación de oro secundario (Pillajo, 2009). Las terrazas aluviales constan principalmente de cantos y bloques redondeados heterolecitos, por lo que es frecuente encontrar terrazas gradadas en diferentes niveles y flanqueando a los depósitos aluviales de los ríos Zamora, Jambüe y Nangaritza.

Mientras que los depósitos coluviales se forman por fenómenos de remoción en masa en zonas de pendiente fuerte y donde hay cambios de pendiente drenan a los ríos principales: Jambüe, Bombuscaro y Nambija, entre otros. Además, se caracteriza por poseer bloques y gravas; ser heterogéneos con fragmentos de rocas mal clasificados y mal trabajados. En estos depósitos, existen deslizamientos activos e inactivos, donde se observa la zona de arranque y la de depósito. Los depósitos aluviales localizados a orillas de los ríos Zamora, Jambüe, Bombuscaro, Nambija, Nangaritza, entre otros. Están constituidos por bloques, cantos rodados, gravas de rocas ígneas intrusivas de composición intermedia a ácida, arenas gruesas y finas (inigemm, 2017) (ver Figura 1).

Se han encontrado indicios de oro en los ríos Mayo, Yunganza, Nambija, Yacuambi, Zamora, Upano, Santiago, San Francisco, Sigsig, Paute, Bomboiza, río Blanco, Mangahurco, La Zarza, Espadillas, Yacu- chingari, Suárez, Machinaza, Collay, Palanda, río Negro, Abanico, Cashpaimi y Tutanangoza. Sin embargo, cabe indicar que la accesibilidad a estos sectores, no es buena, a pesar de tener buenas posibilidades para colocar el equipo necesario para realizar una explotación minera a mediana y gran escala (Pillajo, 2009).

En la recuperación aluvial de oro a bajo costo y para procesar un alto volumen de mineral se utiliza concentradores tipo «Z». Estos concentradores gravimétricos poseen canalones colocados a un ángulo entre los 11 y 30 grados de inclinación, según lo explican varios autores. Esta inclinación depende del tipo de material de alimentación. Los materiales más arcillosos requieren de menos gradiente que los materiales más gruesos. Para la inclinación de los canalones se puede tomar de referencia la inclinación de las mesas de concentración gravimétrica que están entre 0 y 10 grados. Finalmente, se puede mejorar la eficiencia del proceso adicionando en serie otros concentradores. Para lo cual se recomienda colocar un canalón primario, seguido de un jigs y, por último, un segundo canalón de las mismas características que el primario (Santos, 2015; Rodríguez, 2019; Navarro, 2017; Tupiza \& Guzmán, 2019).

Las dimensiones de los canalones son muy variadas, oscilando entre 4,5 y 5,4 $\mathrm{m}$ de largo y $0,54 \mathrm{~m}$ de ancho. En la minería ilegal o antitécnica se adiciona mercurio a las canaletas y/o directamente al concentrado obtenido de las bayetas para la extracción de oro (Rodríguez, 2019; Tupiza y Guzmán, 2019).

Frente al proceso de amalgamación, existen alternativas más limpias para la recuperación del oro de este sector, los concentradores gravimétricos que separan los minerales de diferente gravedad específica por el movimiento a favor de la gravedad y por otra fuerza resistente al movimiento que puede ser un fluido como el agua o el aire. Para conocer de alguna manera el tipo de separación tomando en cuenta la gravedad específica se utiliza el criterio de concentración (Wills y Finch, 2016).

Criterio de concentración. Se utiliza como herramienta matemática para tener una idea general de la facilidad para separar minerales mediante técnicas gravimétricas. Fue desarrollado por Taggart en 1945, sobre la base de datos experimentales (Navarro, 2017; Heredia, 2015). La ecuación (1) representa al criterio de concentración.

Criterio de concentración $=\frac{D h-D l}{D l-D f}$ Ec. (1) 
Tabla 1. Plantas procesadoras in situ para la recuperación de oro secundario

\begin{tabular}{lc}
\hline Minera & Código \\
\hline Sociedad pequeños mineros de San Carlos & SPMSC \\
Sociedad minera de San Carlos & SMSC \\
Sociedad minera de San Carlos Frente 3 & SMSCF3 \\
Sociedad minera de San Carlos Frente 1 & SMSCF1 \\
ENAMI EP & ENAMI \\
Tsamaraint & TSA \\
Solórzano 1 & SOL1 \\
Solórzano 2 & SOL2 \\
Torres & TOR \\
\hline
\end{tabular}

Donde:

$\mathrm{Dh}=$ Densidad relativa del mineral denso

$\mathrm{Dl}=$ Densidad relativa del mineral liviano

Df $=$ Densidad relativa del medio fluido

Cuando el criterio de concentración es mayor a 2,5 la separación es eficiente hasta una malla \#200. Un valor entre 2,5 y 1,75 indica que la separación es eficiente hasta una malla \#100. De 1,75 a 1,50 la separación es posible hasta una malla \#10, valores menores indican que la separación es muy poco eficiente (Navarro, 2017).

Esta investigación está orientada en el estudio del diagnóstico y funcionamiento de la recuperación de oro fino $(-44 \mu \mathrm{m})$ y grueso $(44 \mu \mathrm{m}$ a $1000 \mu \mathrm{m})$ en la minería aluvial con el uso de concentradores tipo $\mathrm{z}$.

\section{METODOLOGÍA}

\section{SELECCIÓN DE LAS MUESTRAS}

Las zonas de interés fueron seleccionadas con base en la relevancia de la actividad de recuperación de oro secundario en el distrito minero. En la tabla 1 se muestran las diferentes plantas procesadoras de recuperación de oro secundario con su respectiva codificación.

En el trabajo de campo se identificaron los principales equipos utilizados en la explotación y beneficios de minerales, además se efectuaron mediciones de las dimensiones de los concentradores gravimétricos como la altura del equipo, altura de la tolva, ancho de canalón, tamaño de luz de la malla del canalón, longitud de parrilla y tamaño de luz de parrilla (ver Tabla 1).

Para la determinación del flujo volumétrico, se consideró la velocidad lineal de la pulpa en función del tiempo de transporte de una pieza plástica en una trayectoria conocida. La medición se realizó 5 veces $y$, además, se midieron el ancho del canalón y altura de la cama de agua para determinar el flujo de descarga, conforme los datos que se requieren para la Ec. (2).

$$
q=\frac{d}{t} \cdot a \cdot h \cdot 3600 \text { Ec. (2) }
$$

Donde:

q: flujo volumétrico de descarga de la pulpa $\left(\mathrm{m}^{3} / \mathrm{h}\right)$ a: ancho del canalón (m)

$\mathrm{d}$ : longitud de la medición (m)

$\mathrm{h}$ : altura de la cama de agua $(\mathrm{m})$

t: tiempo promedio del transporte de la pieza plástica (s)

Para la recolección del mineral de cabeza se procedió de la siguiente manera: se tomaron pequeñas muestras del montículo de material que ingresaba al concentrador, se realizó una homogenización y un cuarteo manual, se escogieron muestras representativas para su caracterización respectiva. Las muestras de relaves se recolectaron utilizando recipientes de 50 l, en los mismos que se hicieron sedimentar los sólidos y se tomó aproximadamente $1 \mathrm{~kg}$ del mineral húmedo.

\section{CARACTERIZACIÓN FISICOQUÍMICA DE LAS MUESTRAS}

Para el análisis granulométrico de las muestras recolectadas en campo, se procedió a secar las muestras en una estufa a una temperatura de $50{ }^{\circ} \mathrm{C}$ y después se realizó un tamizado mecánico en húmedo con la utilización de una gama de tamices que iban de la malla astm \#18 a malla astm \#25, con lo cual se determinó así el diámetro de partícula $\left(\mathrm{dp}_{80}\right)$. 


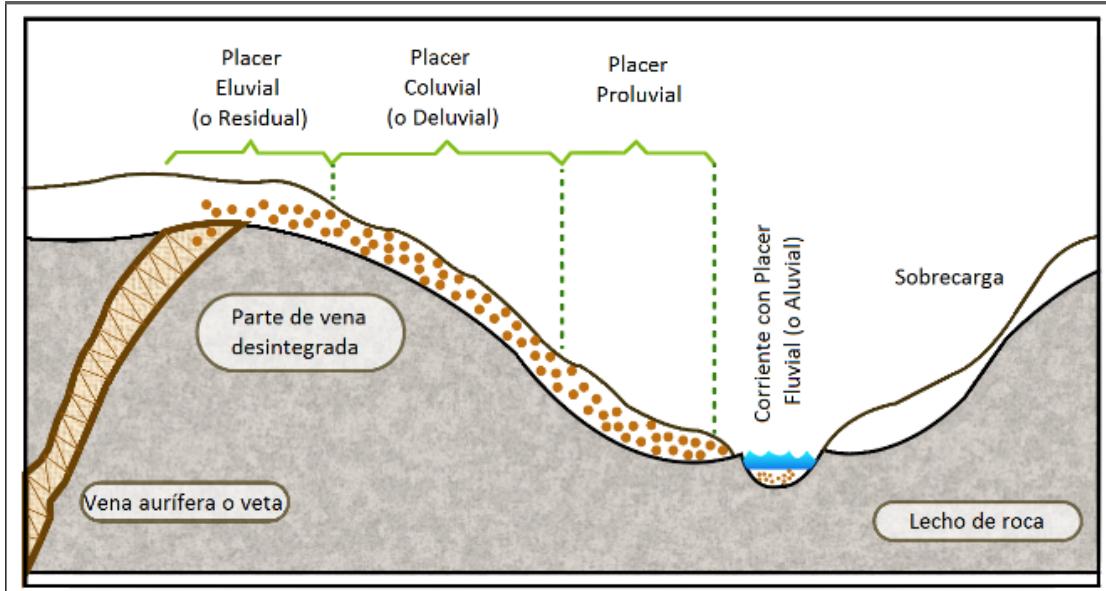

Figura 1. Proceso de formación de placeres aluviales de oro (Marsden y House, 2006).

Para la determinación de oro se utilizó el ensayo al fuego combinado con la lectura en ICP-OEs. La medición de mercurio se realizó por la técnica de generación de hidruros por lectura en un equipo de absorción atómica.

\section{RESULTADOS}

En la figura 2 se observa el esquema $(2 \mathrm{~A})$ y la instalación del equipo concentrador tipo $\mathrm{z}(2 \mathrm{~B})$. El concentrador tipo zeta consiste en dos o más canalones ubicados uno a continuación del otro soportados en una estructura metálica, la misma que está asentada en una base que le proporciona estabilidad. Sus principales componentes son:

- Tolva de alimentación,

- Parrilla para clasificación de mineral,

- Dos o más canalones en serie.

Mientras que los componentes auxiliares de la zeta son:

- monitor de agua a presión,

- una bomba centrífuga,

- sistemas de mangueras para transportar agua desde el río o tanque reservorio,

- excavadora de brazo largo para la alimentación del material. (Ver Figura 2).

La tolva de alimentación se encuentra en la parte más alta de la zeta y tiene una inclinación de $8^{\circ}$ con respecto al eje horizontal, una altura de 60 a 79 $\mathrm{cm}$, ancho de $120 \mathrm{a} 180 \mathrm{~cm}$ y una longitud de $220 \mathrm{a}$ $310 \mathrm{~cm}$. Su capacidad es de un metro cúbico aproximadamente y recibe la alimentación mediante una excavadora de brazo largo. El material es lavado mediante un flujo de agua a presión proporcionado por el monitor para propiciar la disgregación del material fino que se encuentra adherido al material grueso libre de valor. De esta manera, el material fino forma una pulpa que inicia el recorrido a través de los canalones. Por otra parte, el material grueso es eliminado mediante la parrilla de clasificación.

La parrilla está formada por una serie de varillas metálicas de 3 a $6 \mathrm{~cm}$ de diámetro, espaciadas en forma paralela con una distancia de 2 a $4 \mathrm{~cm}$. La parrilla tiene un largo de 2,5 a $3,5 \mathrm{~m}$, y un ancho de 1,2 a 1,5 $\mathrm{m}$ (ver Figura 3). El material que tiene un tamaño inferior a $4 \mathrm{~cm}$ pasa por la abertura de las varillas de la parrilla y continúa hacia los canalones, mientras que el material con un tamaño superior a los $4 \mathrm{~cm}$ es eliminado del sistema.

El canalón consta de un plano de $1,5 \mathrm{~m}$ de ancho y 3,5 $\mathrm{m}$ de largo aproximadamente con una inclinación de $14^{\circ}$ de inclinación con respecto al eje horizontal. Sobre la superficie inclinada del canalón se tiene una malla metálica de una pulgada de alto $(2,54 \mathrm{~cm}), \mathrm{y}$ con un entramado en forma de rombo de $4 \times 8 \mathrm{~cm}$ de luz. Este entramado permite la retención del material pesado en las fibras de yute que se colocan en la parte inferior, lo cual permite la sedimentación del material pesado durante su trayecto por los canalones. En la figura 4 se muestra un esquema de un canalón (ver Figura 4).

Los minerales comunes en los depósitos aluviales son la magnetita, ilmenita, granate y colombo-tantalita y los metales preciosos como oro, plata, y metales del grupo del platino.

En el esquema de la figura 5 se identifica el uso de excavadoras tanto para las operaciones de arranque de la grava aurífera como para la tolva de alimentación del equipo de concentración gravimétrica tipo zeta. La tasa de alimentación registrada para estas actividades se encuentra entre una a tres paladas por minuto, lo cual implica una alimentación de 60 a 180 $\mathrm{m}^{3} / \mathrm{h}$ (ver Figura 5).

La figura 6 muestra los porcentajes de oro fino y grueso de material de alimentación al concentrador $\mathrm{z}$, se observa que de las nueve plantas mineras 
Tabla 2. Flujos de descarga de pulpa y descargas de mercurio

\begin{tabular}{lcc}
\hline Minera & Flujo descarga $(\mathbf{m} \mathbf{3} / \mathbf{h})$ & Descarga $\mathbf{H g} \mathbf{( g / \text { ton} )}$ \\
\hline ENAMI & 88 & $<0,01$ \\
TSA & 49,7 & $<0,01$ \\
SOL1 & 174,6 & $<0,01$ \\
SOL2 & 172,2 & 1,75 \\
TOR & 84,6 & 2,57 \\
\hline
\end{tabular}

Fuente: s. d.

de Zamora, cinco de éstas presentan un porcentaje mayor al $60 \%$ oro fino en el material alimentado a la zeta mientras que dos presentan resultados con mayor fracción de oro grueso y una presenta alrededor de la mitad de fino y grueso; teniendo los resultados más destacados para la empresa tor con $98 \%$, sol1 $90 \%$, sol2 $80 \%$, enami $83 \%$, smscf1 $78 \%$ y smsc $60 \%$ de oro fino, mientras se tiene que la empresa smscf3 con $88 \%$ y spmsc $64 \%$ de oro grueso. Finalmente, la empresa que está alrededor del $50 \%$ entre oro fino y oro grueso es tsa con $42 \%$ y $58 \%$ respectivamente. La concentración gravimétrica no es recomendable para las partículas menores a $50 \mu \mathrm{m}$ puesto que la eficiencia de la operación disminuye (ver figura 6).

En la figura 7 se muestra el porcentaje de oro fino y grueso del material de descarga del concentrador z; se observa cuatro empresas que descargan oro fino, entre ellas están: smsc con $95 \%$, enami y tsa con $95 \%$ y soll con $96 \%$ mientras que las empresas que descargan oro grueso son sPMSc con $65 \%$, smscf3 con $96 \%$, smscf1 con $88 \%$ y sol 2 con $64 \%$ y una empresa descarga alrededor del $50 \%$ es tor con $46 \%$ de oro fino y $54 \%$ con oro grueso. Lo que se observa es que cuatro concentradores $\mathrm{z}$ no recuperan oro fino, cuatro no recuperan oro grueso y una está entre $50 \%$ de oro fino y grueso.

En la figura 7 se muestra el porcentaje de oro en fracciones fino y grueso en la descarga del concentrador tipo zeta, cinco empresas presentan un porcentaje mayor del $50 \%$ de oro grueso, por ejemplo, smscf 3 con $96 \%$, smscf1 con $88 \%$, spmsc con $65 \%$ sol2 con $64 \%$ y tor con $54 \%$. En las otras cuatro empresas se observa oro fino como son: soll con $96 \%$, smsc, enami y tsa con 95\% (ver Figura 7).

Al evaluar los resultados del tamaño de oro de las descargas se encontró que las operaciones que presentan porcentajes de recuperación de oro grueso son tsa con $54 \%$, smsc con $35 \%$, enami $12 \%$, y sol1 con $6 \%$ mientras que las empresas que recuperan oro fino son smscf1 con $66 \%$, tor con
$52 \%$, sol2 44\% y smscf3 con $8 \%$. La empresa spmsc presenta la más baja recuperación de oro fino con tan solo $1 \%$.

La tabla 2 muestra los flujos de descarga de pulpas de las empresas mineras y el contenido de mercurio descargado. Se encontró que la Compañía Minera Solórzano 2 presenta un contenido de mercurio en los sólidos de la descarga de $1,75 \mathrm{mg} / \mathrm{kg}$ y para la Sociedad Minera Torres es de 2,57 mg/kg. Estos resultados evidencian la utilización del mercurio paralelamente a la operación de concentración gravimétrica. Por otro lado, las empresas que trabajan con mayor flujo son sol1 y sol2 con un alrededor del 173 $\mathrm{m} 3 / \mathrm{h}$ mientras enami y tor trabajan con un flujo alrededor de $85 \mathrm{~m} 3 / \mathrm{h}$ y, finalmente, la empresa tsa con flujo de $49,7 \mathrm{~m} 3 / \mathrm{h}$, menor a las anteriores empresas (ver Tabla 2).

Al evaluar los resultados del concentrado tipo zeta de las nueve empresas con la recuperación de oro, flujo de descarga de la pulpa y la descarga de mercurio se tiene lo siguiente. La empresa tsa con un porcentaje de recuperación de oro grueso de $54 \%$, un flujo de $49,7 \mathrm{~m} 3 / \mathrm{h}$ y con una descarga de mercurio de menor a $0,01 \mathrm{~g} /$ ton. Luego está la empresa enami con $12 \%$ de recuperación de oro grueso, con un flujo de $88,0 \mathrm{~m} 3 / \mathrm{h}$ y una descarga de mercurio menor a 0,01 $\mathrm{g} /$ ton. soll recupera $6 \%$ de oro grueso con un flujo de $174,6 \mathrm{~m} 3 / \mathrm{h}$ y una descarga de mercurio menor a $0,01 \mathrm{~g} /$ ton. Por otro lado, las empresas que recuperan oro fino son tor con $52 \%$, un flujo de $84,6 \mathrm{~m} 3 / \mathrm{h}$ y una descarga de mercurio de $2,57 \mathrm{~g} /$ ton y sol 2 con un porcentaje de recuperación de $44 \%$, un flujo de 172,2 $\mathrm{m} 3 / \mathrm{h}$ y con un contenido de mercurio de $1,75 \%$.

\section{DISCUSIÓN DE RESULTADOS}

Cuanto menor es el flujo de descarga en un concentrador tipo zeta se tiene mayor recuperación de oro grueso sin uso de mercurio, como lo demuestra la empresa TSA que recupera $54 \%$ de oro grueso con un menor flujo equivalente a $49,7 \mathrm{~m}^{3} / \mathrm{h}$. En contraste, 

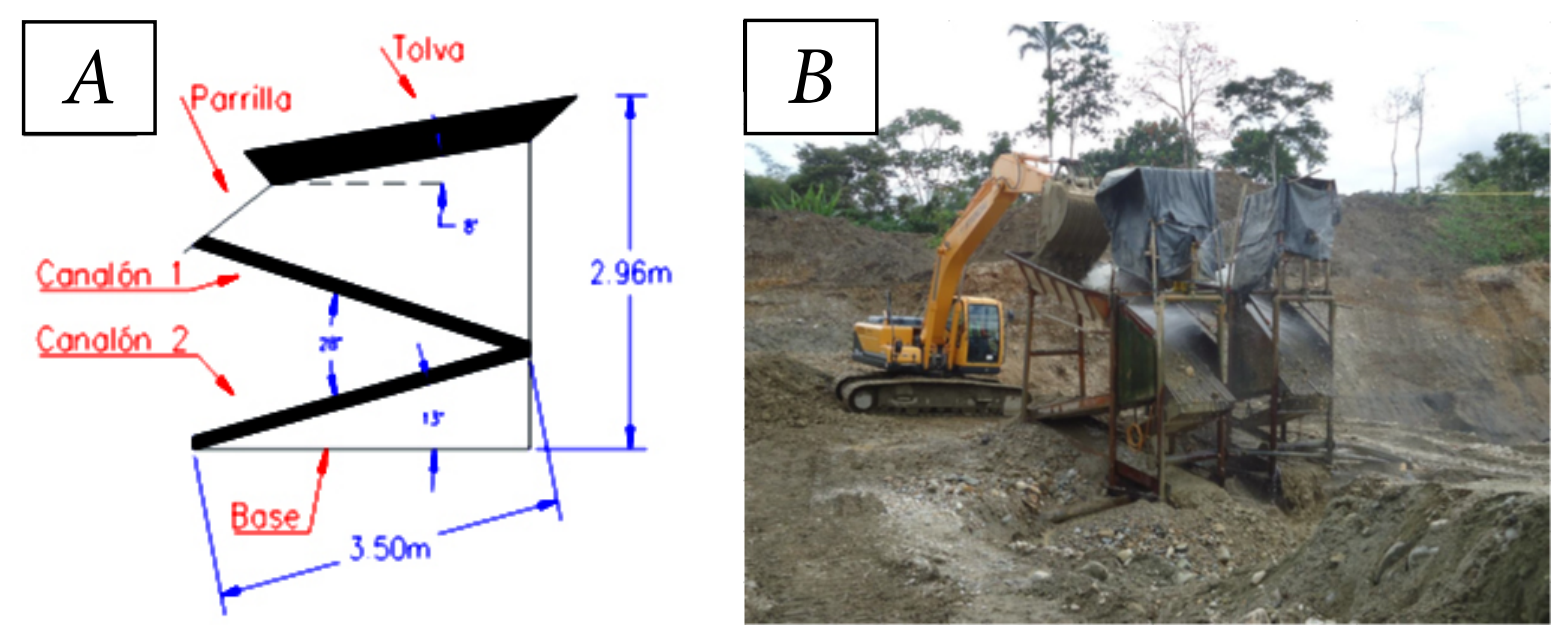

Figura 2. A) Esquema de un concentrador gravimétrico tipo Zeta. B) Instalación para recuperación de oro secundario en la Sociedad Minera Solórzano 2.

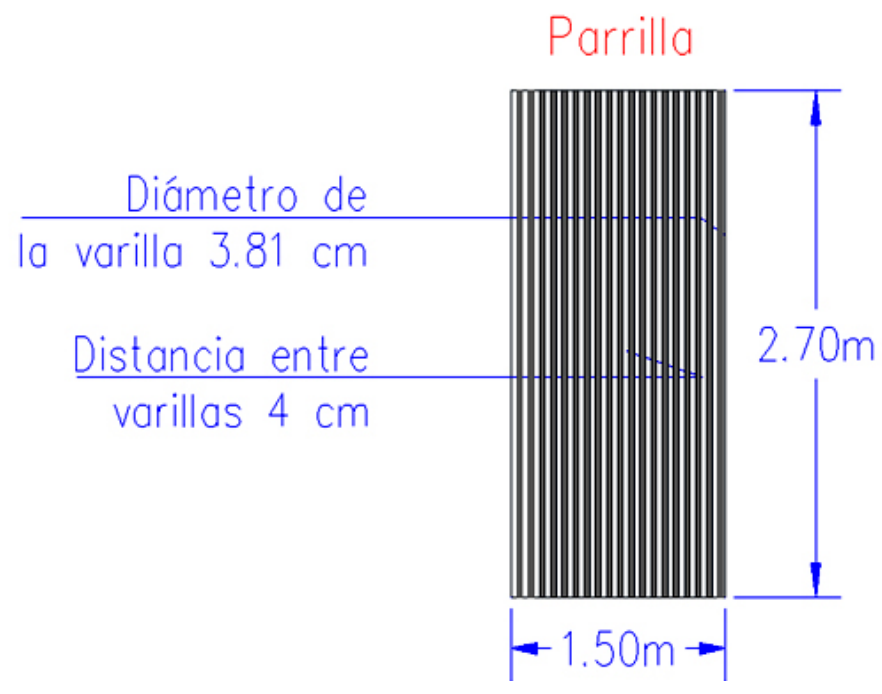

Figura 3. Parrilla para recolectar oro

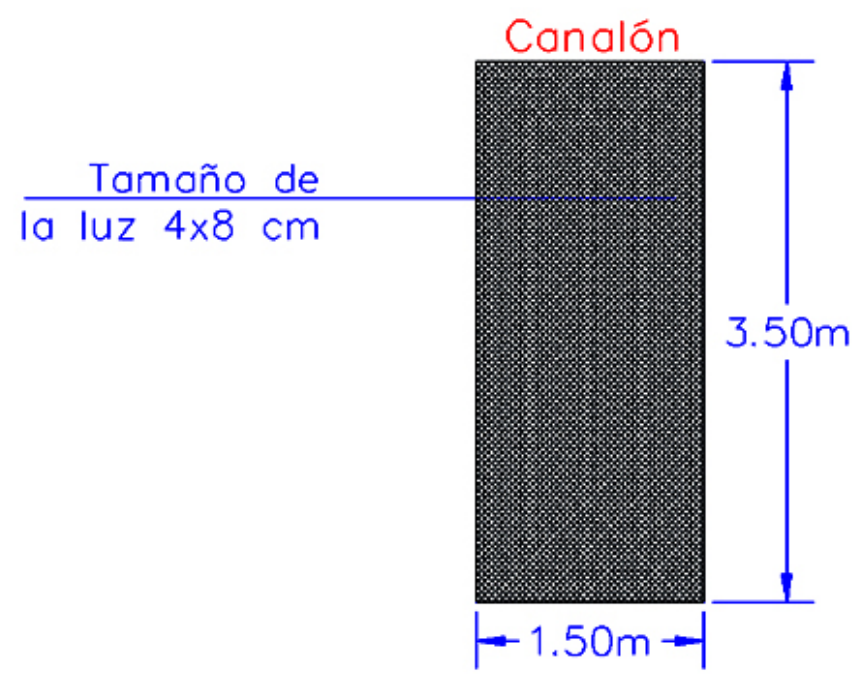

Figura 4. Canalón para recolectar oro 


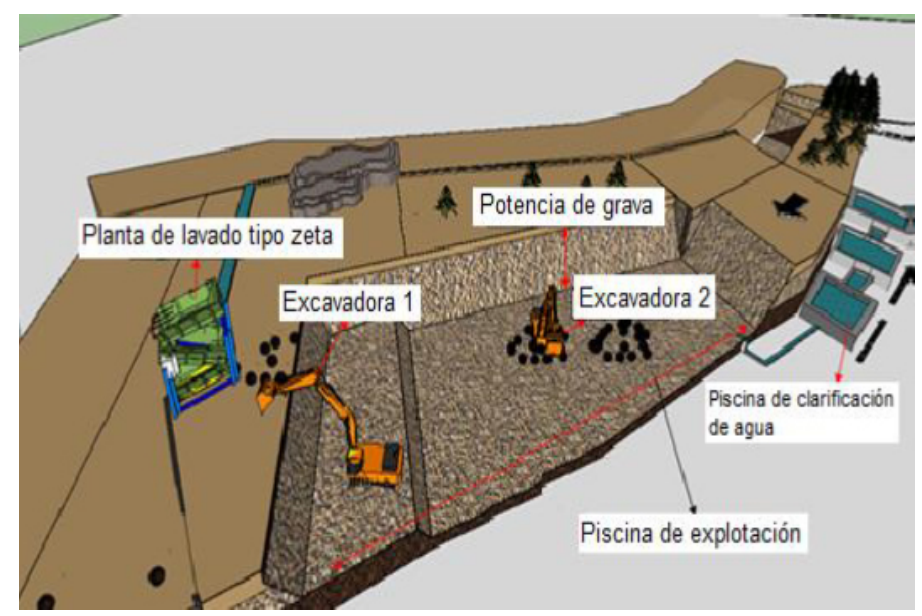

Figura 5. Procesamiento y equipamiento utilizado en la extracción de oro aluvial en el distrito minero de Zamora (Zúñiga, 2013)

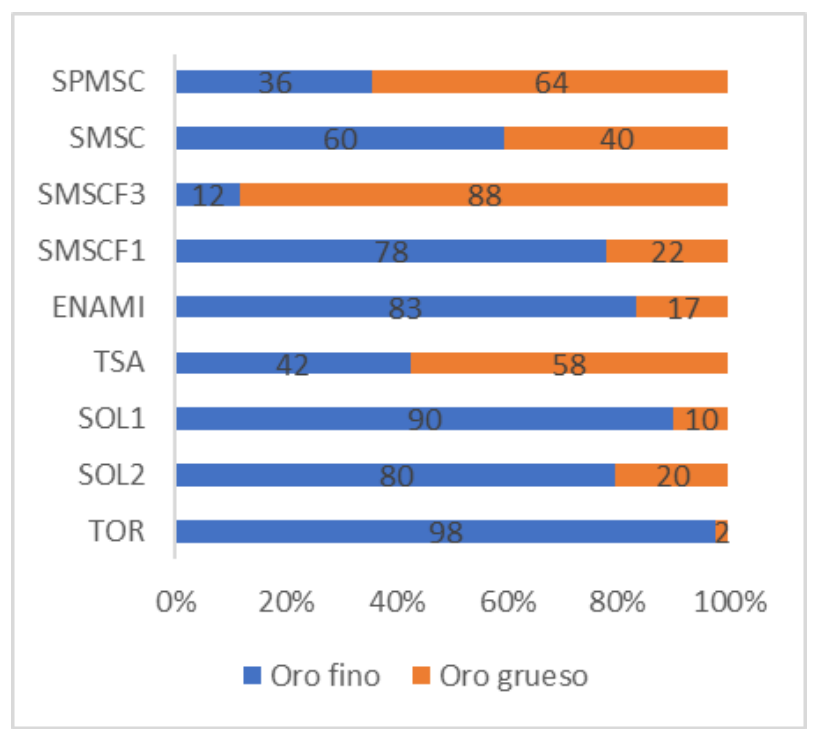

Figura 6. Porcentaje de oro en fracciones en el material alimentado a la zeta.

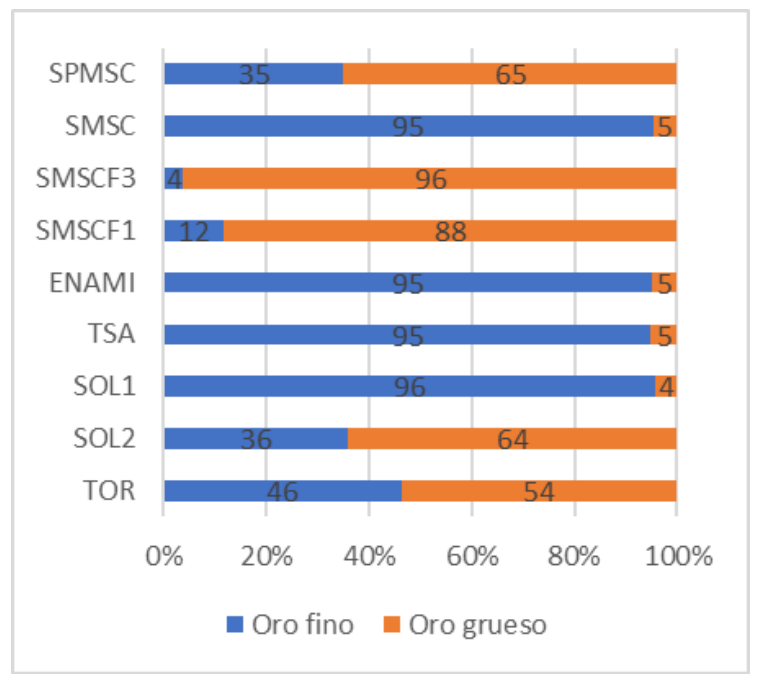

Figura 7. Porcentaje de oro en fracciones en el material descargado del concentrador tipo zeta. 
la empresa sol1 con un flujo mucho mayor a la TSA $174 \mathrm{~m}^{3} / \mathrm{h}$ recupera menor cantidad de oro grueso de aproximadamente el 6\%. Es concluyente decir que al trabajar con mucho más flujo se está favoreciendo la pérdida de oro grueso.

Por otro lado, la recuperación de oro fino se ve favorecido por el uso de mercurio en el concentrador tipo $\mathrm{z}$, ya que las empresas que presentan recuperación de oro fino tienen flujos que van entre 84 a 174 $\mathrm{m}^{3} / \mathrm{h}$.

Se puede decir que a menor flujo de descarga se puede recuperar oro grueso como lo demuestra la empresa TSA, con el menor flujo obtiene la mayor recuperación. Mientras que a mayor flujo decae la recuperación de oro grueso, además provoca que nada de oro fino se recupere.

En conclusión, el uso del concentrador tipo z no es nada eficiente en la recuperación de oro grueso como en la recuperación de oro fino.

\section{CONCLUSIONES}

Los procesos de extracción metalúrgica de oro aluvial en el distrito de Zamora se encuentran limitados, ya que la maquinaria utilizada es ineficiente en comparación con los equipos utilizados en la concentración gravimétrica convencional. Debido a los altos grados de inclinación que utiliza el concentrador tipo z, la eficiencia de recuperación del oro grueso se ve desfavorecida. Por otra parte, los procesos de refinación de los concentrados de oro son perjudiciales al medio ambiente, ya que se utiliza mercurio como colector de oro y, a su vez, no se les da un tratamiento a los materiales descargados, causando una contaminación de los cuerpos de aguas superficiales próximos a la extracción metalúrgica.

En la búsqueda de alternativas amigables con el ambiente y eficientes en la recuperación de oro en los concentradores $\mathrm{z}$, se deberían tomar en cuenta el criterio matemático como el criterio de concentración TAGART, con el fin de determinar la facilidad de separación de minerales a partir de la concentración gravimétrica.

Los concentradores z deberían utilizar el principio de funcionamiento de los canalones teniendo en cuenta el ancho con relación a la cantidad de agua que se cuenta; el material de retención debería ser de alfombras de rizos y contar con ángulos de inclinación entre $5^{\circ}$ y $15^{\circ}$.
Para el beneficio del oro fino perdido en las colas de los concentradores gravimétricos se debería estudiar la utilización de concentradores centrífugos para la recuperación del oro no aprovechado.

\section{REFERENCIAS}

Heredia, Y. 2015. Estudio para el montaje y puesta en marcha de una planta para el beneficio del mineral de oro en la zona de reserva especial minera del Municipio de Quinchía Risaralda. Universidad Tecnológica de Pereira, Pereira.

INIGEMM. 2017. Hoja geológica Zamora. http://www. geoenergia.gob.ec/mapas-geologicos/.

Marsden, J., y House, I. 2006. The chemistry of gold extraction (2. ${ }^{\text {a }}$ ed.). Littleton, USA: Society for Mining, Metallurgy, and Exploration, Inc.

Navarro, M. 2017. Estudio de la recuperación de oro aluvial en concentrador falcon en la cooperativa minera Limata Ananea-Puno. Universidad Nacional Jorge Basadre Grohmann-Tacna, Tacna.

Pillajo, E. 2009. Evaluación del potencial aurífero aluvial en Ecuador. http://fungeomine.org/documentos/potencial_aurifero_aluvial_en_el_ecuador.pdf

Rodríguez, R. 2019. Ampliación de proceso bonanza e instalación de planta Rumicuri-Ecuador. Universidad Nacional de San Agustín de Arequipa, Arequipa.

Santos, D. 2015. Diseño de explotación de la grava aurífera existente en el área minera Durán (código 300050), Santa Rosa-El Oro. Universidad Central del Ecuador, Quito, Ecuador.

Stewart, J., Kerr, G., Prior, D., Halfpenny, A., Pearce, M., Hough, R., y Craw, D. 2017. Low temperature recrystallisation of alluvial gold in paleoplacer deposits. Ore Geology Reviews, 88, 43-56.

Tupiza, J. y Guzmán, A. 2019. Tecnologías limpias para el aprovechamiento de depósitos detríticos (oro aluvial) ubicados en la provincia del Napo (Tesis de grado). Universidad Central del Ecuador, Quito, Ecuador.

Wills, B., Finch, J. 2016. Wills' mineral processing techno$\log y$ (8. ${ }^{a}$ ed., cap. 10). Gravity Concentration, pp. 223244.

Zuñiga, C. 2013. Diseño de explotación del depósito aurífero aluvial del río Cachaví, frente San Antonio, de la Empresa Nacional Minera ENAMI E.P. (Tesis de grado). Universidad Central del Ecuador, Quito, Ecuador. 